\title{
Heterogeneous nucleation and crystal growth on curved surfaces observed by real-space imaging
}

\author{
F Ziese $^{1}$, G Maret $^{1}$ and U Gasser ${ }^{2}$ \\ ${ }^{1}$ Physics Department, University of Konstanz, D-78457 Konstanz, Germany \\ ${ }^{2}$ Laboratory for Neutron Scattering, Paul Scherrer Institut, 5232 Villigen PSI, Switzerland \\ E-mail: urs.gasser@psi.ch
}

\begin{abstract}
We present a real-space imaging study of homogeneous and heterogeneous crystal nucleation and growth in colloidal suspensions of slightly charged and polydisperse particles.

Heterogeneous crystallization is observed close to curved surfaces with radii of curvature, $R$, in the range from 4 to 40 particle diameters, $d$. Close to a curved surface, we find crystal nucleation and growth to be suppressed for $R \lesssim 10 d$. For $R \gtrsim 15 d$, fast crystal growth is observed similar to that on a flat wall $(R=\infty)$. We use the purely topological method of shortest path rings to determine the orientation of the crystal on the length scale of the nearest neighbor distance. Crystal nuclei forming close to a curved surface are oriented analogous to crystal growth on a flat wall with hexagonal planes parallel to the wall. While the smallest nuclei appear to be unaffected by the surface, larger nuclei are found to be suppressed for radii of curvature $R \lesssim 10 \mathrm{~d}$. The critical nucleus size in the vicinity of a curved surface is found to be about the same as for homogeneous nucleation.
\end{abstract}

\section{Introduction}

Crystal nucleation has been studied for more than 100 years and is of importance for the fundamental understanding of phase transitions, material properties and manufacturing. Nevertheless, there are still important open questions regarding the process of crystal nucleation. Crystal nucleation is a first-order phase transition and, therefore, there is no general theory, and the nature of the transition depends on the studied material. Furthermore, the properties of microscopic and mesoscopic crystal nuclei forming due to structural fluctuations in a supercooled fluid are not well known. There is evidence that small crystal nuclei may not have the same structure [1] and surface tension [2] as the bulk crystal. However, classical nucleation theory assumes that these nuclei can be described using the properties of the bulk crystal [3]. As a consequence, experimental as well as theoretical progress in the understanding of small crystal nuclei is needed. It is well accepted that crystal nucleation is governed by the free energy barrier

$$
\Delta G_{\mathrm{hom}}=\gamma_{\mathrm{cf}} A-\Delta \mu n V
$$

giving the free energy change due to the formation of a crystal nucleus with surface area $A$ and volume $V . \gamma_{\text {cf }}$ is the surface tension between the crystal and the fluid, $\Delta \mu$ is the chemical potential difference of the metastable fluid and the crystal phase, and $n$ is the particle number density in the crystal. The dependence of $\gamma_{\mathrm{cf}}$ and $\Delta \mu$ on the size, shape, and structure of the crystal nucleus is, however, not known in detail. Furthermore, $\Delta G$ can be increased or decreased in the presence of impurities or boundaries of the system, depending on the values of the surface tension of the crystal, $\gamma_{\mathrm{cs}}$, and the fluid, $\gamma_{\mathrm{fs}}$, on the imposed surface. When $\Delta G$ is comparable to or becomes lower than $\Delta G_{\text {hom }}$, the impurity can act as a seed for crystal nucleation and heterogeneous nucleation takes place on the seed, which can be much faster 
than homogeneous nucleation in the bulk of a supercooled fluid. The free energy barrier for heterogeneous nucleation is given by

$$
\Delta G_{\mathrm{het}}=\gamma_{\mathrm{cf}} A_{\mathrm{cf}}+\left(\gamma_{\mathrm{cs}}-\gamma_{\mathrm{fs}}\right) A_{\mathrm{cs}}-\Delta \mu n V+\tau L .
$$

$\tau$ is the line tension of the contact line defined by the co-occurrence of seed, fluid, and crystal, and $L$ is the length of this line. Computer simulations of hard spheres next to a flat, hard wall (of infinite radius of curvature) show that a small free energy barrier exists for crystal nucleation on the wall [4]. This barrier is mainly given by the line tension $\tau$, and hexagonal planes of hard spheres are found to be close to spontaneously wetting the wall. On the other hand, seed particles with radii of curvature from two to five times the hard sphere diameter are found to hinder the formation of crystal nuclei. As a consequence, there must be a transition from a high to a low barrier in $\Delta G$ with increasing radius of curvature of the seed particles. Moreover, computer simulations suggest that seed particles in an optimal size range promote the formation of critical crystal nuclei that can overcome the barrier in $\Delta G$ and detach from the seed particle to become large crystal grains [5]. In this way, seed particles may function as 'catalysts' for crystal nucleation.

The properties of microscopically small crystals in a fluid are hard to study in atomic or molecular systems, as their size is $\sim 1 \mathrm{~nm}$ and they evolve in a timescale shorter than $1 \mu \mathrm{s}$. Colloidal particles are $\sim 10^{3}$ times larger and typical timescales are beyond seconds to minutes, which makes them experimentally much more accessible. Therefore, colloidal suspensions have become an important test bed to study crystal nucleation with single particle resolution and in the timescale of the formation of crystal nuclei [2, 6, 7]. In particular, modern laser scanning confocal microscopy has become an important tool for the study of local structures such as crystal nuclei in colloidal suspensions $[8,9]$.

\section{Experimental details}

We study both homogeneous and heterogeneous crystal nucleation as well as crystal growth in suspensions of poly(methyl methacrylate) (PMMA) spheres [10, 11], which are labeled with fluorescein to make them visible with fluorescence microscopy. Their average diameter is $d=1.9 \pm 0.05 \mu \mathrm{m}$ and the polydispersity is $0.067 \pm 0.005$ as determined from dynamic light scattering with CONTIN analysis [12]. The spheres are suspended in a mixture of cyclo-heptylbromide and decahydronaphthalene, which nearly matches the density and the refractive index of the particles and, therefore, allows for observations of the particles as deep as $100 \mu \mathrm{m}$ from the sample surface.

The radial pair-distribution functions (see supporting material available at stacks.iop.org/JPhysCM/25/375105/ mmedia) determined for fluid samples with volume fractions $\phi=0.036$ and 0.049 are compatible with a hard-sphere Yukawa interaction [13, 14] with a screening length of $330 \pm 20 \mathrm{~nm}$ and a contact value of $42 \pm 5 k_{\mathrm{B}} T$, where $k_{\mathrm{B}} T$ is the thermal energy. This implies a surface charge $\approx 380 e$ of the particles, where $e$ is the elementary charge.
At $\phi=0.134$, a comparison yields the same screening length, but a higher contact value of $127 \pm 5 k_{\mathrm{B}} T$ is found. This significant change with $\phi$ indicates the importance of many-body forces in the studied suspensions, as expected from [14] reporting on a similar colloidal system. The effect of many-body forces becomes apparent at higher volume fractions. Therefore, a contact value of $42 \pm 3 k_{\mathrm{B}} T$ gives the strength of the interaction, but many-body forces are not negligible at $\phi \gtrsim 0.05$. We observe freezing at $\phi_{\mathrm{f}}=0.16 \pm 0.01$ and fluid-crystal coexistence up to the melting point at $\phi_{\mathrm{m}}=0.20 \pm 0.02$. This agrees well with the phase behavior reported for hard-sphere Yukawa particles in [13], where the freezing line for a contact value of $39 k_{\mathrm{B}} T$ and a screening length of $330 \mathrm{~nm}$ is found at $\phi=0.18$.

Samples with a thickness of $0.5 \mathrm{~mm}$ are prepared in glass cells with a microscopy cover glass on one side. For the study of homogeneous nucleation, crystal growth on the cell walls is suppressed with a rough and irregular coating. This is done by sticking polydisperse PMMA particles with a mean diameter of $6.5 \mu \mathrm{m}$ onto the walls by heating them up to $130^{\circ} \mathrm{C}$, slightly above the glass temperature of PMMA. Heterogeneous nucleation is probed near glass spheres with radii from 10 up to $70 \mu \mathrm{m}$, which are located at the bottom of the cells due to sedimentation. Suspensions are imaged on a Zeiss Axiovert 200 inverted microscope with a Yokogawa Nipkow disk scanner, an oil-immersion objective with numerical aperture 1.45, and a field of view of up to $87 \times 66 \mu \mathrm{m}^{2}$. The microscope objective is mounted on a piezo scanner, allowing the focal plane to be moved in the range 0-90 $\mu \mathrm{m}$ from the cover slip. Typically, stacks of about 120 images are taken with steps of $200 \mathrm{~nm}$ from image to image for the observation of about $10^{4}$ particles in $3 \mathrm{~d}$. Crystal nucleation and growth are followed by taking many image stacks for up to $70 \mathrm{~h}$. The time step between image stacks is chosen between $20 \mathrm{~s}$ and $20 \mathrm{~min}$, depending on the desired time resolution.

The image stacks allow a determination of the coordinates for all visible particles in $3 \mathrm{~d}$. This is done with a sub-pixel resolution of $\approx 20 \mathrm{~nm}$ according to the method of Crocker and Grier [15]. Uneven illumination in the images is corrected, and a real-space bandpass filter suppresses pixel noise and enhances the visibility of the features with the size of the particles. Local brightness maxima are chosen as potential particle positions, and the final particle coordinates are obtained from a brightness-weighted centroid fit [15].

For the detection of small crystal nuclei in fluid surroundings, the arrangement of particles needs to be analyzed on a local scale. Local bond order parameters based on the spherical harmonic functions $Y_{l m}$ are, therefore, used to obtain a quantitative measure of the arrangement of the neighbors for each particle. Bond order parameters with $l=4$ and 6 have been introduced for the characterization of local order in glasses [16] and they have been used previously to identify small crystal nuclei $[1,17,2,18]$. The local bond order parameter with $l=6$ for particle $\alpha$ is given by

$$
q_{6 m}(\alpha)=\frac{1}{N(\alpha)} \sum_{\beta=1}^{N(\alpha)} Y_{6 m}\left(\hat{r}_{\alpha \beta}\right),
$$




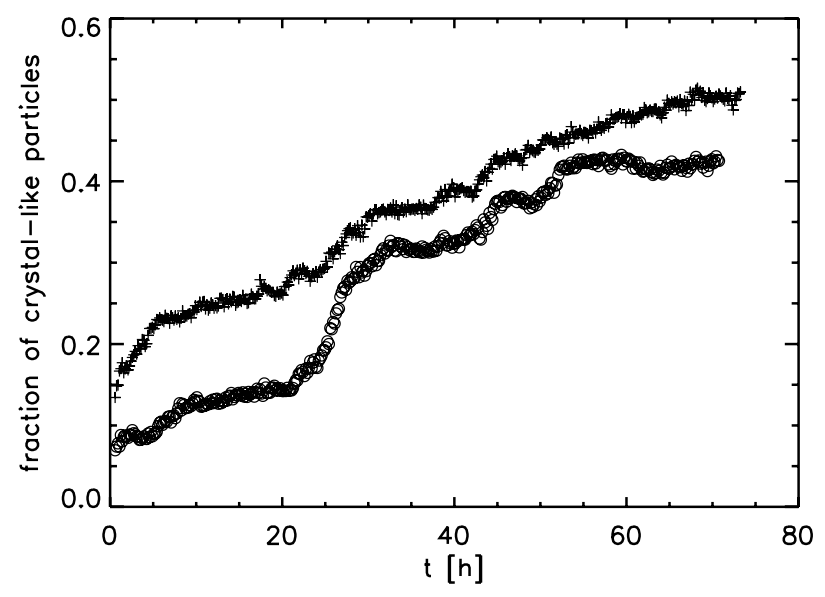

Figure 1. Fraction of crystal-like particles as a function of the time after shear-melting at $t=0$ for two measurements of homogeneous crystallization at $\phi=0.29$.

where $\hat{r}_{\alpha \beta}$ gives the direction from particle $\alpha$ to one of its $N(\alpha)$ nearest neighbors, which are defined by a cut-off distance chosen such that on average 12 nearest neighbors are obtained, as expected for the crystal observed in the system studied. As the arrangement of neighbors is the same for each particle in a perfect crystal, the $q_{6 m}$ are expected to be similar for all particles in a crystal nucleus; this contrasts with a fluid surrounding, where the arrangement of neighbors varies quickly from particle to particle. If the inner product of the normalized $\hat{q}_{6 m}=q_{6 m} / \sqrt{\sum_{m} q_{6 m} q_{6 m}^{*}}$ of two neighboring particles $\alpha$ and $\beta$ exceeds a threshold of 0.5 , they are counted as particles connected by a crystal-like bond ${ }^{3}$ :

$$
\sum_{m=-6}^{6} \hat{q}_{6 m}(\alpha) \hat{q}_{6 m}^{*}(\beta)>0.5
$$

We call particles with a crystal-like arrangement of neighbors crystal-like particles. These are identified by searching for particles with eight or more crystal-like bonds to their next neighbors. Crystal nuclei can be found by identifying clusters of crystal-like particles that are connected by crystal-like bonds.

To keep track of crystal nuclei over time, the nuclei identified in consecutive snapshots are compared based on their spatial overlap and their averaged bond order parameters

$$
\bar{q}_{6 m}(i, t)=\frac{1}{N(i, t)} \sum_{\alpha=1}^{N(i, t)} q_{6 m}(\alpha, t),
$$

where $i$ denotes the nucleus containing $N(i, t)$ particles at time $t$. The averaged bond order parameters are compared using the scalar product $c_{q_{6}}(i, t ; j, t+1)=\sum_{m} \hat{\bar{q}}_{6 m}(i, t) \hat{\bar{q}}_{6 m}^{*}(j, t+1)$, analogous to equation (4). The spatial overlap, $c_{\text {overlap }}$, is defined as the number of particles having a 'neighbor' in the other nucleus within a distance of $d_{\mathrm{n}}=2.5 \mu \mathrm{m}$ divided by the

\footnotetext{
3 Bonds between neighboring particles are based on a given distance
} criterion only and are not related to chemical bonds averaged nucleus size:

$$
\begin{aligned}
c_{\text {overlap }}= & \frac{2}{N(i, t)+N(j, t+1)} \\
& \times \sum_{k=1}^{N(i, t)} \sum_{l=1}^{N(j, t+1)} \Theta\left(d_{\mathrm{n}}-|\boldsymbol{r}(k, t)-\boldsymbol{r}(l, t+1)|\right),
\end{aligned}
$$

where $\Theta$ is the Heaviside function. Finally, the sum of the two criteria has to fulfil the condition that $c_{\text {overlap }}+c_{q_{6}}>1$ and $c_{\text {overlap }}>0.3$ to accept two nuclei as predecessor and successor. In case several nuclei at time $t+1$ fulfil this criterion, the nucleus with the highest $c_{\text {overlap }}+c_{q_{6}}$ is selected as the successor. For tracks that start or end because no successor or predecessor can be assigned, nuclei giving a sum $c_{\text {overlap }}+c_{q_{6}}<1$ or being assigned to another nucleus are counted as possible successors or predecessors.

\section{Results and discussion}

\subsection{Crystal-like particles}

To gain insight into the nucleation and growth phase using confocal microscopy, we have taken measurements quickly after shear-melting the sample and focused on the early times when critical nuclei form. As the formation of a critical nucleus that continues to grow is a rare event, our measurements only allow for a rough estimate of the critical size. However, the formation of precritical nuclei that approach the critical size are found in all measurements and crystal growth into the observed area from the outside is common. The number of crystal-like particles as a function of time is shown in figure 1 for two examples of homogeneous crystal growth. The formation of precritical crystal nuclei is found to start quickly, but crystal growth is slow and is still ongoing after $\sim 70 \mathrm{~h}$. An induction time for crystal nucleation is expected [19], however the polydispersity of the particles further slows down the nucleation process. From computer simulations of hard spheres [20], it is well known that crystallization does not occur in suspensions with polydispersity $\gtrsim 0.12$, while the differences from a monodisperse system are small for polydispersities $<0.05$. Furthermore, the polydispersity in the crystal is found to be always below 0.057. A demixing of small and large particles has to take place in the suspensions studied here, because the polydispersity is $\approx 0.067$. In agreement with this, we observe a slow crystallization process: the formation of precritical nuclei is found to start quickly within $\sim 10 \mathrm{~min}$, but it takes $\sim 10$ days until the equilibrium phase behavior of crystal coexisting with fluid or complete crystallization is reached. Furthermore, we observe incomplete crystallization even at $\phi>\phi_{\mathrm{m}}$, as expected for the polydispersity of the studied suspensions. This agrees with previous observations that bidisperse or polydisperse hard spheres do not crystallize at all or do so more slowly than in the monodisperse case [20-22]. A slow crystallization process is also observed close to the glass transition and for the devitrification of glassy samples [23]. This may play a role for the most concentrated 

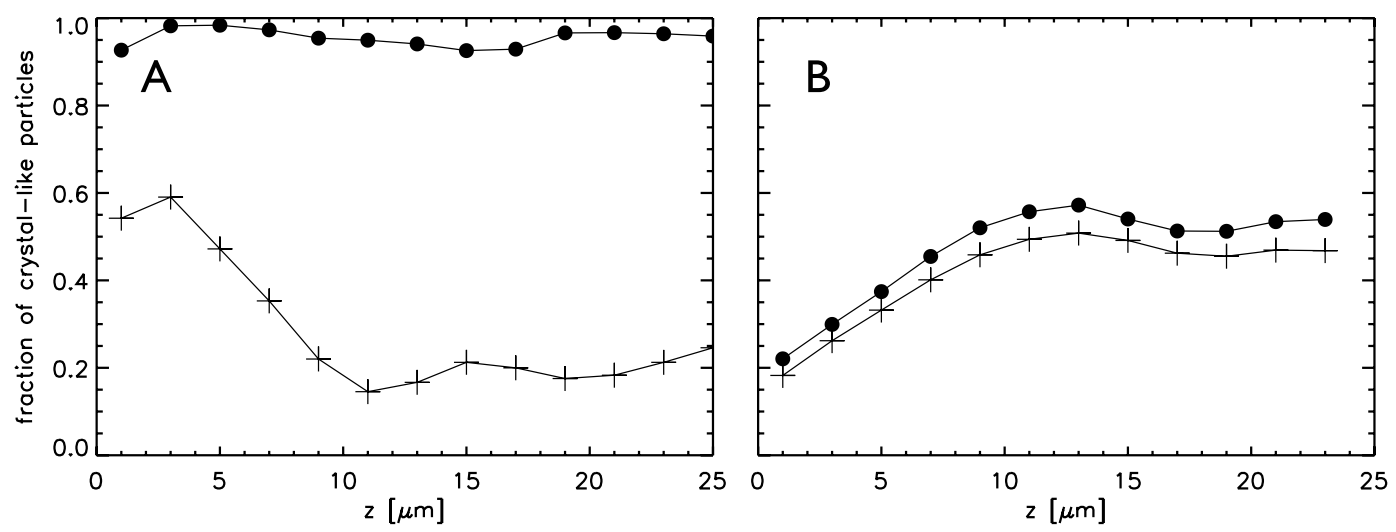

Figure 2. Fraction of crystal-like particles as a function of the distance, $z$, from (A) a flat and (B) a rough surface. The fractions are shown for $(+) 20 \mathrm{~min}$ and $(\bullet) 20 \mathrm{~h}$ after shear-melting the sample. The average height of the rough surface is at a distance of $\approx-2 \mu \mathrm{m}$. The volume fractions are (A) $\phi=0.25$ and $(\mathrm{B}) \phi=0.29$.

samples studied in this work. In the following we concentrate on the early stage of nucleation and growth within $\sim 48 \mathrm{~h}$ after shear-melting the sample.

\subsubsection{Flat and rough surfaces. To quantify the influence of} a surface or a particle as a seed for crystallization, we count the crystal-like particles as a function of the distance from the seed. The cases of a flat and a rough surface are studied for comparison. The flat surface enhances the fraction of crystal-like particles near the surface, as shown in figure 2(A), since there is no or a very small free energy barrier and the crystal grows spontaneously on the surface. The condition for this spontaneous crystal formation is $\gamma_{\mathrm{fs}}-\gamma_{\mathrm{cs}}>\gamma_{\mathrm{cf}}$, which appears to be fulfilled in the studied colloidal system for an infinite radius of curvature, $R=\infty$. The rough surface suppresses crystal nucleation close to the surface, and the number of crystal-like particles near the surface is therefore diminished, as shown in figure 2(B). The rough coating of the wall is obtained with polydisperse PMMA spheres with an average diameter $\approx 3.5 \mathrm{~d}$. The suppression of crystal growth close to the rough wall shows that the free energy barrier close to the wall is at least as high as in the bulk. This suppression of crystal growth on the wall of the sample cell provides an optimized environment for the study of nucleation and growth in the bulk or on seed particles in the suspension, which can be much slower than the growth on a flat surface. As shown in figure 3 , the roughness also suppresses the layering of the particles that is usually observed next to a wall [24]. This layering also reflects the spontaneous crystallization next to a flat wall and the reduced influence of a curved wall.

3.1.2. Curved surfaces. Six examples of curved surfaces with different radii of curvature are shown in figure 4 . The percentage of crystal-like particles near the surface is reduced for radii of curvature $R \lesssim 10 d$, is about the same as in the bulk for $10 d \lesssim R \lesssim 13 d$ and becomes larger than in the bulk for $R \gtrsim 15 d$. Therefore, the radius of curvature has a clear effect on crystal nucleation and growth. Our observations suggest that the free energy barrier is reduced for radii of curvature $R \gtrsim 15 d$. The curved surface appears to strongly affect the formation of crystal-like structures within a distance $\approx 5 d \approx 10 \mu \mathrm{m}$, which roughly corresponds to the range with layered particle structure (see figure 3 ).

\subsection{Crystal orientation}

Crystallization of hard and slightly charged spheres on a flat surface is known to take place spontaneously with hexagonal crystal layers forming parallel to the surface, as there is no nucleation barrier. However, surfaces with a radius of curvature $R \sim 5 d$ are found to hinder crystal nucleation. As a consequence, there is a transition from hindrance to spontaneous crystal growth when the radius of curvature is increased. This suggests that curved surfaces influence the orientation of the crystal that forms close-by. Bulk crystal in our system is found to be built of hexagonal layers with a stacking corresponding to a face centered cubic (fcc) and hexagonal close packed (hcp) crystal as reported in the literature $[25,26,2,14,27,13]$. The normal vector for these hexagonal planes can be used to quantify the orientation of the crystal. As illustrated in figure 5, the $(0,0,1)$-plane is the only hexagonal plane in the hcp crystal lattice, while the $(1,1,1)-$, $(1,1,-1)-,(1,-1,1)-$, and $(-1,1,1)$-planes are the four equivalent hexagonal planes in the fcc lattice. As no large scale hexagonal planes exist in small crystal nuclei, it is useful to look for hexagonal planes defined by a single particle and its nearest neighbors. The 'rings' of six particles highlighted in figure 5 can be used for this purpose and can be identified using the method of shortest path rings that analyzes a set of particle positions and the bonds between them on the basis of purely topological criteria [28-30]. We apply this method to the nearest neighbors of a central particle and the bonds between them, ignoring the central particle and its bonds. A shortest path ring is a ring of particles with the property that there is no shorter path from one ring particle to another than the path along the ring; here, all the bonds between all particles are considered to find a shortcut. The length of the path is measured by counting the number of bonds that are passed. In the fcc lattice, each particle has four shortest path rings of six particles (6-rings), and these correspond to 

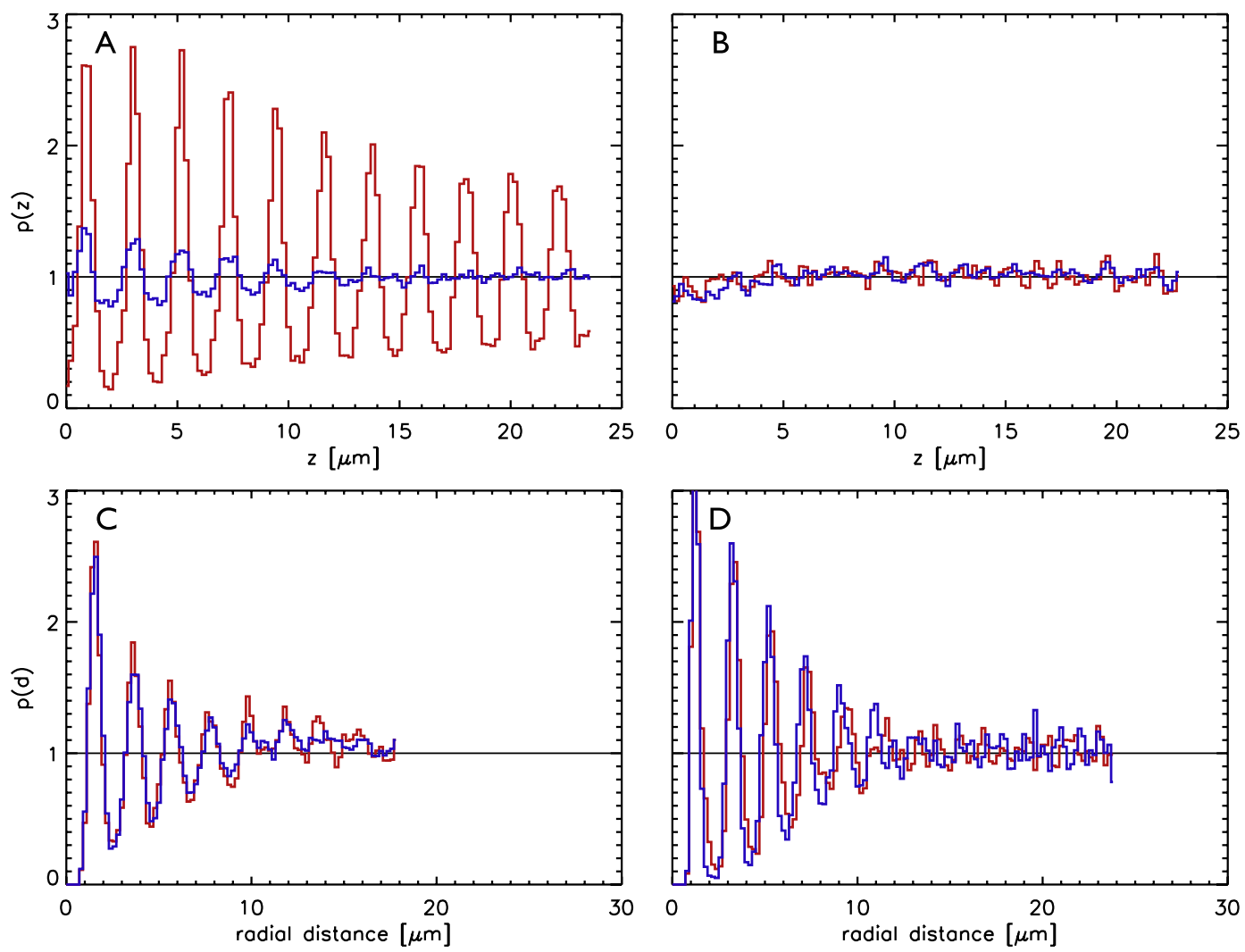

Figure 3. Measured particle densities normalized by the average density as a function of the distance from a flat wall (A), a rough wall (B), and curved surfaces with radii of curvature $R=18.3 \mu \mathrm{m}(\mathrm{C})$ and $29.8 \mu \mathrm{m}(\mathrm{D})$. The density is given for $\sim 2 \mathrm{~h}$ (blue) and $\sim 48 \mathrm{~h}(\mathrm{red})$ after shear-melting. The volume fractions are $\phi=0.24$ (A), $\phi=0.29$ (B), (C), and $\phi=0.31$ (D).

hexagonal planes of the lattice. As for fcc, each particle in the hcp lattice has four 6-rings. However, just one belongs to a hexagonal plane and the other three are buckled and not related to hexagonal planes (figure 5(B)). These buckled 6-rings can be sorted out using topological information only: For each 6-ring, the particles not belonging to the 6-ring (among the nearest neighbors of the studied particle) are grouped in clusters according to their bonds. There are two groups of three particles for a 6-ring corresponding to a hexagonal plane, while a group with two and a group with four particles are found for the buckled rings, as shown in figure 5(B). For the detection of hexagonal planes, the shortest path ring method can also be applied to particles with 11 or 13 instead of the expected 12 nearest neighbors. The treatment of these exceptions is important for measurements of small crystal nuclei without a well-defined crystal structure. In these cases, 6-rings characterizing hexagonal planes are found using additional criteria, as outlined in the appendix. 6-rings belonging to particles with less than 11 or more than 13 nearest neighbors are not taken into account, as they correspond to strongly disturbed crystal structures. In the following, buckled 6-rings are disregarded and only 6-rings belonging to potential hexagonal planes are taken into account. A plane is fit to each 6-ring to obtain its orientation and that of a corresponding hexagonal plane.

3.2.1. Flat and rough surfaces. As expected, a flat wall given by the cover glass of the sample cell results in a strong orientation of the 6-rings close to it. This is shown in figure $6(\mathrm{~A})$ by the strong peak of the distribution of orientations, $p(\theta) / \sin (\theta)$, at the tilting angle $\theta=0^{\circ}$ for all measured distances in the range $0-25 \mu \mathrm{m}$ from the surface. This reflects the growth of hexagonal planes on a flat surface immediately after shear-melting. $\theta$ is defined as the angle between the direction normal to the flat surface and the direction normal to the plane of the 6-ring. The histograms shown are normalized by $\sin (\theta)$, as the number of possible orientations at a tilting angle $\theta$ is proportional to $\sin (\theta)$ in analogy to the area of a ring on a sphere at a fixed angle $\theta$ from the pole. The normalization is chosen such that a fully random distribution of orientations corresponds to $p(\theta) / \sin (\theta)=1$. For a fcc crystal, there are additional 6-rings belonging to the three other hexagonal planes, and they show up at a tilting angle $\theta \approx 70.5^{\circ}$. A broad peak is indeed observed at $\theta \approx 70^{\circ}$ for the distances $0-20 \mu \mathrm{m}$; however it appears not to be symmetric around $70^{\circ}$ but to be skewed towards smaller $\theta$. A broadening is expected due to fluctuations of the particle positions, and the peak is more pronounced at smaller $\theta$, because the normalization by $\sin (\theta)$ emphasizes orientations at small angles. In fully crystallized samples, the number of 6-rings at $\theta \approx 70^{\circ}$ is found to be roughly three times larger than that at $\theta=0^{\circ}$, as expected for a fcc crystal. For distances 20-25 $\mu \mathrm{m}$ (magenta points in figure 6(A)), 6-rings with an orientation $\theta \approx 60^{\circ}$ are detected during early times for this particular measurement; these belong to precritical crystal nuclei that do not grow. 

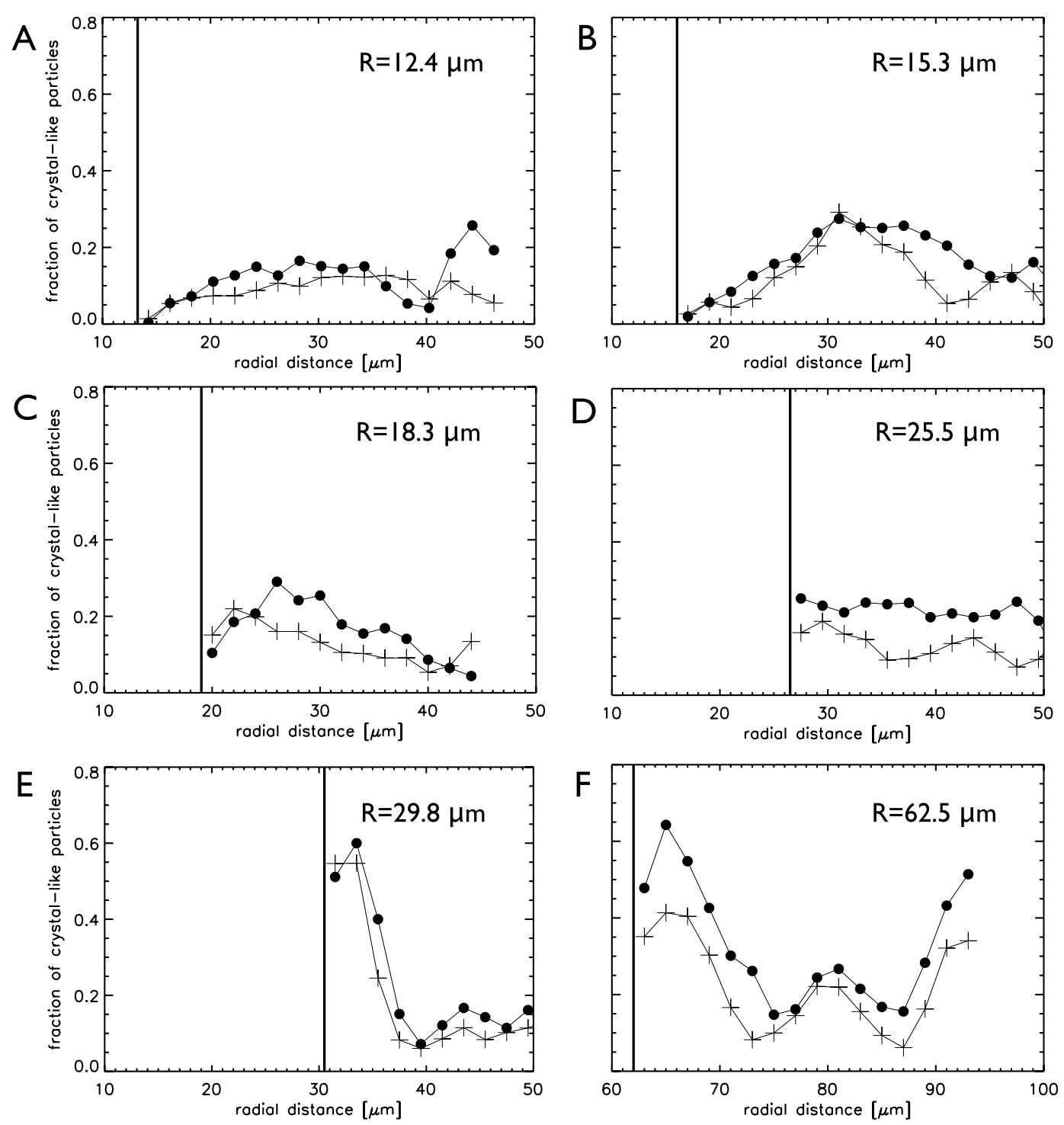

Figure 4. Fraction of crystal-like particles as a function of the distance from the center of the curved surface for the radii of curvature (A) $R=12.4 \mu \mathrm{m}$, (B) $15.3 \mu \mathrm{m}$, (C) $18.3 \mu \mathrm{m}$, (D) $25.5 \mu \mathrm{m}$, (E) $29.8 \mu \mathrm{m}$, and (F) $62.5 \mu \mathrm{m}$. The radii of curvature are indicated by the black vertical lines. The volume fractions are (A) $\phi=0.27$, (B), (C) $\phi=0.29$, (D) $\phi=0.28$, (E) $\phi=0.31$, and (F) $\phi=0.24$. The symbols show the fraction obtained $20 \mathrm{~min}(+)$ and $20 \mathrm{~h}(\bullet)$ after shear-melting the sample.

The crystal oriented parallel to the flat surface is found to keep growing until the observed volume is filled with oriented crystal. On the other hand, the rough surface described above suppresses crystallization on the wall and the orientation of 6-rings of crystal-like particles is found to be random, as shown in figure 6(B). All curves for the distances $0-25 \mu \mathrm{m}$ fluctuate around $p(\theta) / \sin (\theta)=1$, the value expected for completely random orientations; variations with $\theta$ are due to precritical crystal nuclei appearing for some time during the measurement. This shows that surfaces with a radius of curvature $R \sim 4 d$ strongly suppress crystallization, which was also found from simulations of hard spheres [5, 31]. The insets of figure 6 show the number of crystal-like particles, $N_{\mathrm{c}}$ (red), and of the corresponding 6-rings, $N_{6}$ (black). We find $N_{\mathrm{c}} \lesssim N_{6}$; the number of observed 6-rings is lower than expected from a 50:50 mixture of particles with local fcc and hcp structure with four and one 6-rings per particle, respectively. This reflects the low order in precritical crystal nuclei.

3.2.2. Curved surfaces. As suggested by the case of a flat surface, we determine whether crystal particles appearing in the vicinity of a curved surface have 6-rings oriented parallel to the surface. The tilting angle $\theta$ is now measured between the normal vector of the 6-ring and the surface normal, and the $\theta$-histograms for six radii of curvature, $R$, between 12 and $70 \mu \mathrm{m}$ are shown in figure 7 . The crystal-like particles with distances from 0 to $10 \mu \mathrm{m}$ from the surface have 6-rings oriented parallel to the curved surface for all investigated radii of curvature. For the shell between 0 and $5 \mu \mathrm{m}$ (red dots), the orientation is very strong, as shown by the peak at $\theta=0^{\circ}$, and a broad peak around $\theta=70^{\circ}$ shows that many crystal-like particles have a fcc-like surrounding. As apparent from the 


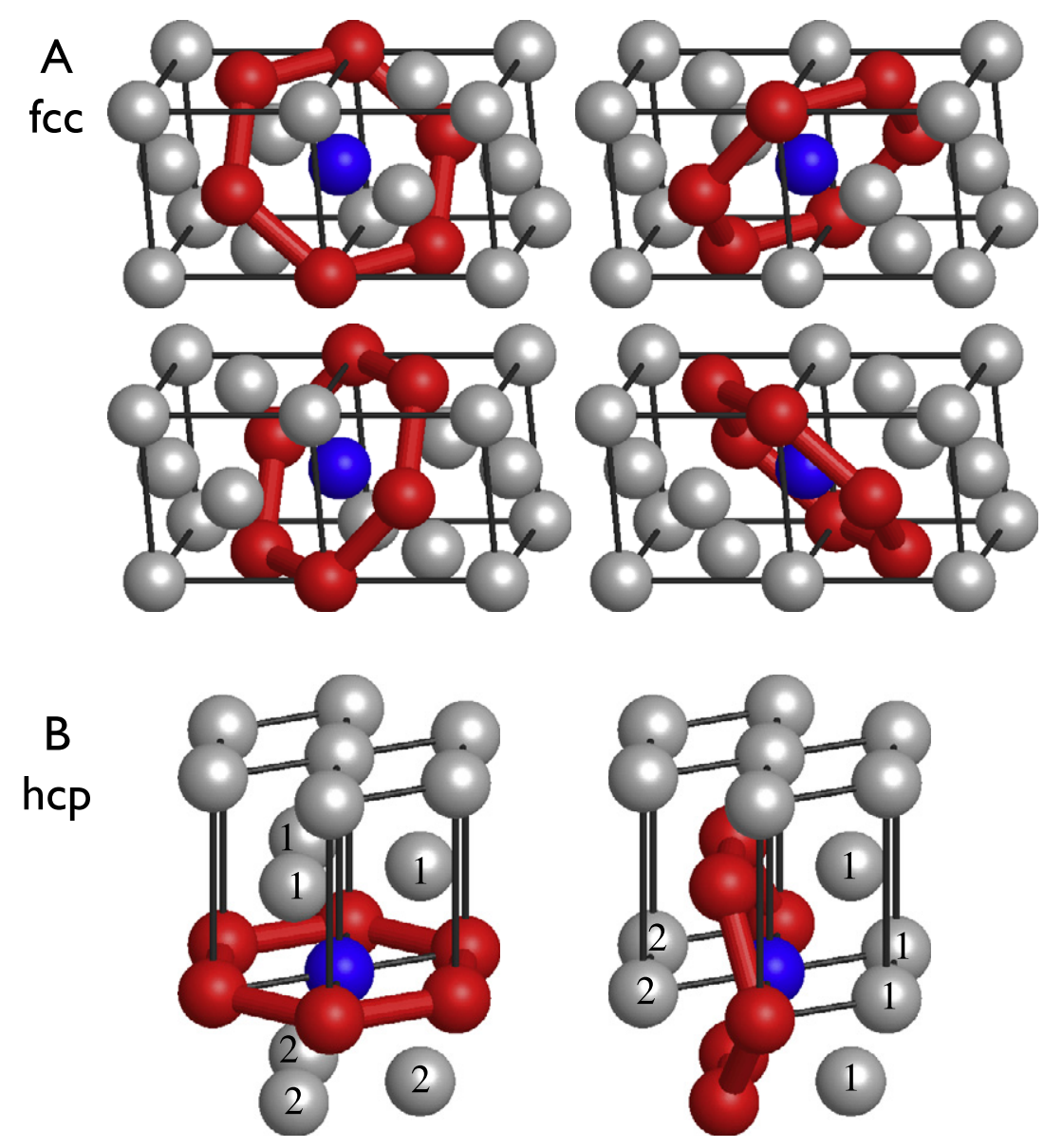

Figure 5. Shortest path rings of six particles (6-rings), highlighted red, around a central particle (blue) in crystal with (A) fcc and (B) hcp structure. (A) In fcc, there are four equivalent 6-rings that belong to hexagonal planes. (B) For hcp crystal, one 6-ring is flat and belongs to a hexagonal plane ((B), left), while the buckled 6-rings are not related to hexagonal planes ((B), right). The flat 6-ring has two groups of three particles on both sides, marked ' 1 ' and ' 2 ', while the buckled rings have a group of four and a group of two neighbors, marked ' 1 ' and ' 2 ', respectively.
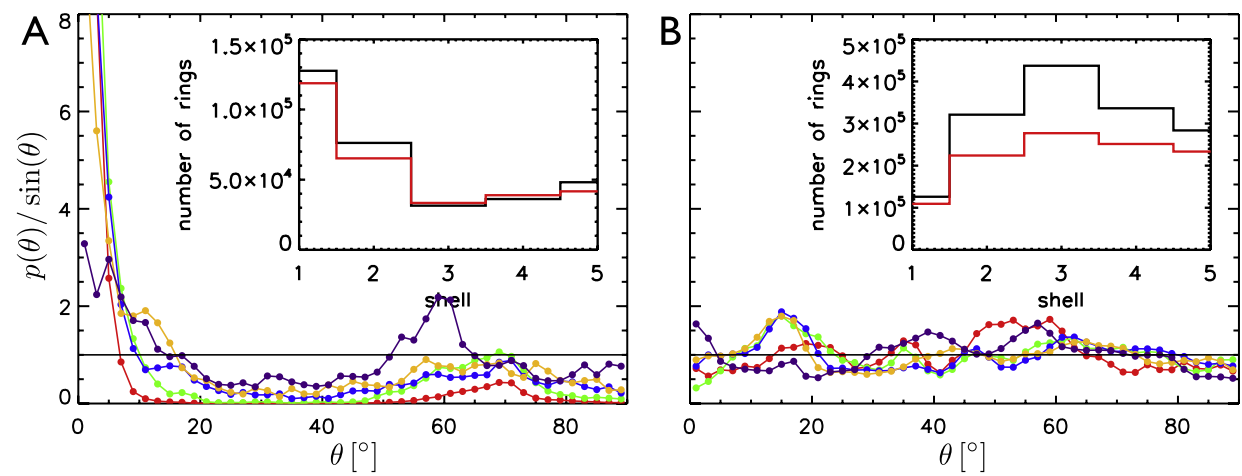

Figure 6. Probabilities for the 6-ring orientation versus tilting angle $\theta$ for (A) a flat and (B) a rough surface. The probability distribution is shown for several distances from the surface: (red, shell 1) 0-5 $\mu \mathrm{m}$, (green, shell 2) 5-10 $\mu \mathrm{m}$, (blue, shell 3) 10-15 $\mu \mathrm{m}$, (yellow, shell 4) 15-20 $\mu \mathrm{m}$, (magenta, shell 5) 20-25 $\mu \mathrm{m}$. The horizontal lines at $p(\theta) / \sin (\theta)=1$ give the value for a random distribution. The insets show the number of (black) 6-rings and (red) crystal-like particles found in each measurement for the different-distance shells.

shell between 5 and $10 \mu \mathrm{m}$ (green dots), the orientation of the 6-rings gets more pronounced for larger radii of curvature. The order in the shells between 10 and $25 \mu \mathrm{m}$ is found to increase for radii of curvature $R \geq 25.5 \mu \mathrm{m}$; the peaks at $\theta \approx 0^{\circ}$ and $70^{\circ}$ get more pronounced with increasing $R$. This orientation over a longer range and the reduced suppression of crystal-like particles close to the surface (figure 4(D)) suggest that the transition from hindered crystallization to accelerated heterogeneous crystal nucleation and growth analogous to that on a flat surface occurs close to $R=13 d \approx 25 \mu \mathrm{m}$. 

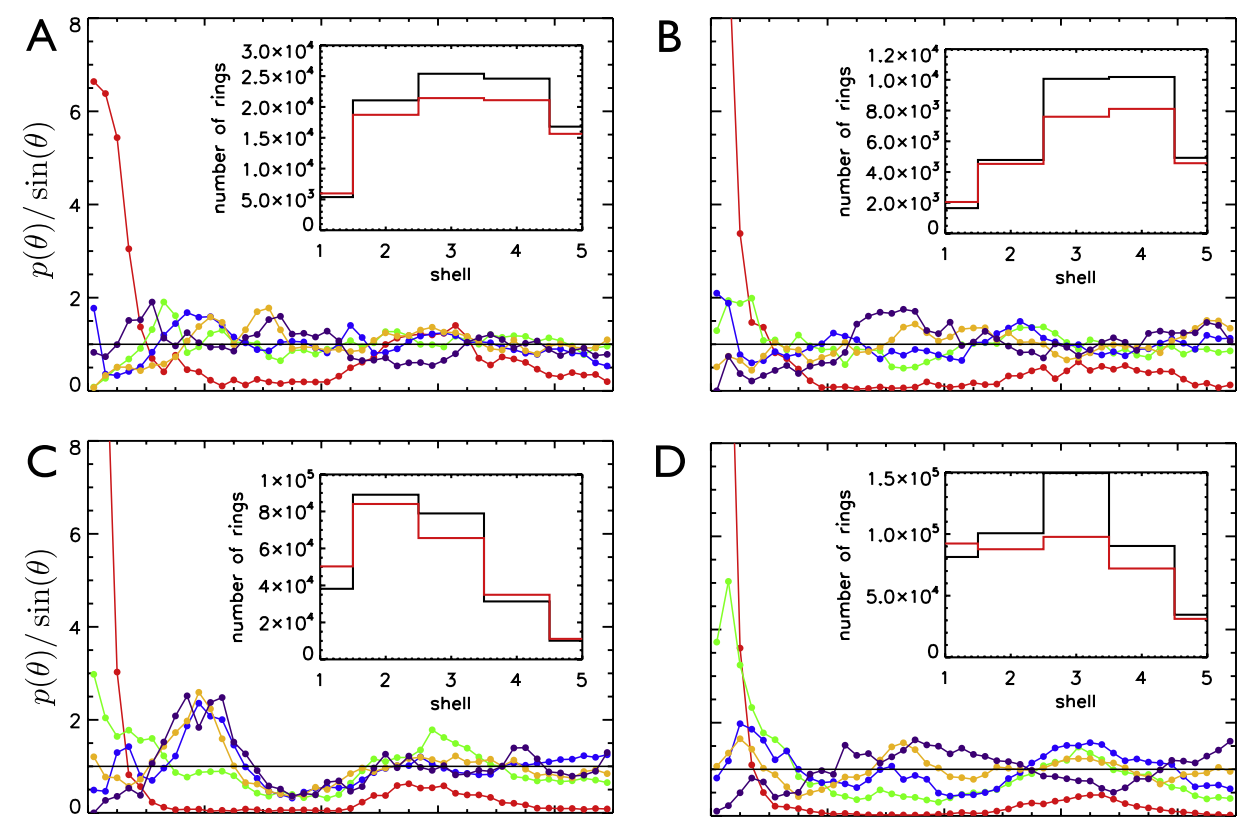

$\mathrm{D}$
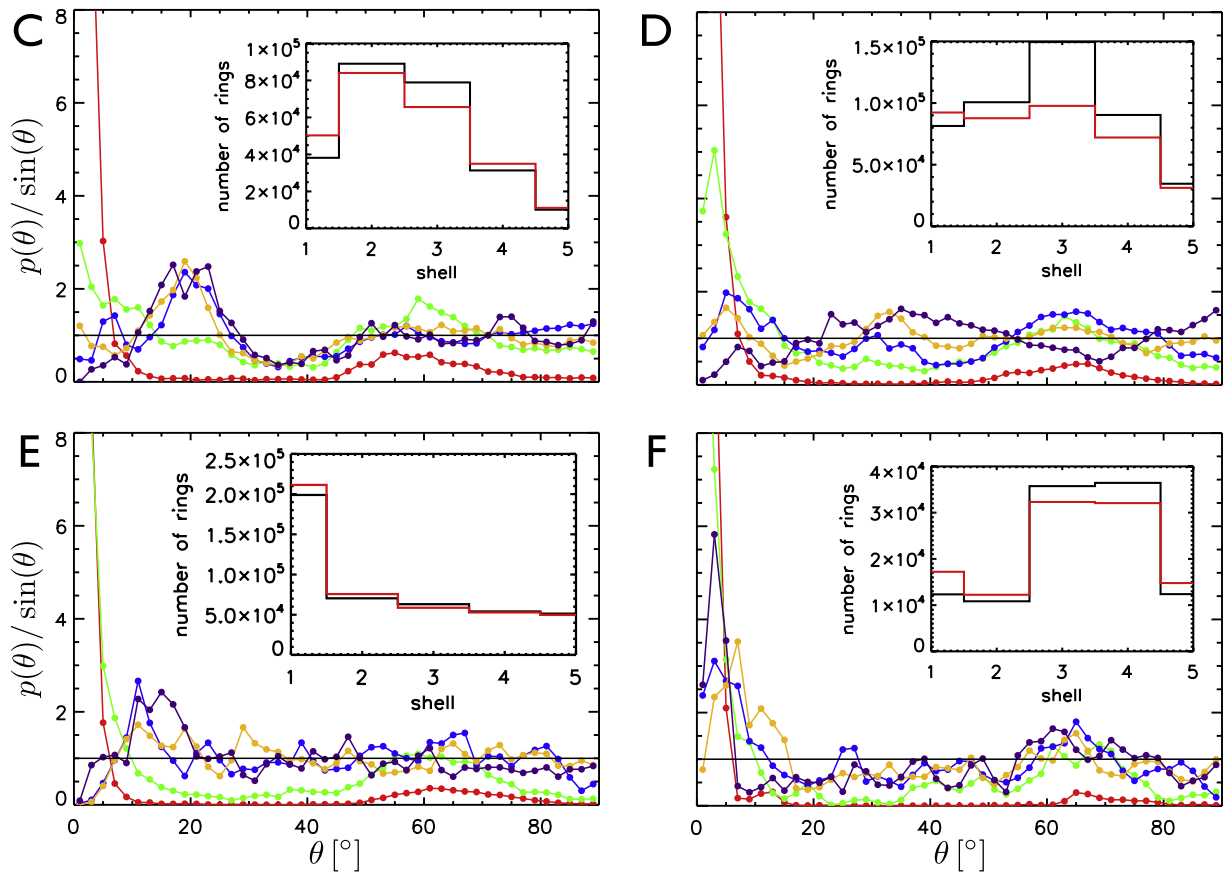

Figure 7. Probabilities for the 6-ring orientation versus tilting angle $\theta$ for the radii of curvature (A) $12.4 \mu \mathrm{m}$, (B) $15.3 \mu \mathrm{m},(\mathrm{C}) 18.3 \mu \mathrm{m}$, (D) $25.5 \mu \mathrm{m}$, (E) $29.8 \mu \mathrm{m}$, and (F) $62.5 \mu \mathrm{m}$. The probability distribution is shown for several distances from the surface: (red, shell 1) 0-5 $\mu \mathrm{m}$, (green, shell 2) 5-10 $\mu \mathrm{m}$, (blue, shell 3) 10-15 $\mu \mathrm{m}$, (yellow, shell 4) 15-20 $\mu \mathrm{m}$, (magenta, shell 5) 20-25 $\mu \mathrm{m}$. The horizontal lines at $p(\theta) / \sin (\theta)=1$ give the value for a random distribution. The insets show the number of (black) 6-rings and (red) crystal-like particles found in each measurement for the different-distance shells. The volume fractions for the measurements shown are the same as those given in figure 4.

\subsection{Precritical crystal nuclei}

The conclusion given above is corroborated by an analysis of precritical nuclei close to the curved surfaces. As the free energy penalty for such nuclei increases with their area and volume (equation (1)), very small nuclei appear much more often than larger ones. We have determined the number of nuclei containing $M<15$ and $M \geq 15$ particles as a function of the distance from curved surfaces, as shown in figure $8 . M$ is the number of particles in a nucleus. The number of observed nuclei, $N_{\mathrm{n}}$, is normalized by the total number of particles, $N_{\mathrm{p}}$, at the same distance to account for the variation of the volumes of the shells with distance. The number of nuclei with $M \geq 15$ particles $(x)$ is found to be strongly suppressed for radii of curvature $R \lesssim 10 d$ and distances $\lesssim 3 d=5.7 \mu \mathrm{m}$ from the surface. Very small nuclei with $M<15(\bullet)$ are always at a level comparable to that in the bulk. However for $R \gtrsim 13 d$, nuclei of all sizes are at least as numerous close to the surface as far from it, as expected for the transition to accelerated nucleation for larger $R$. For $R \approx 13 d$, crystal growth on the surface appears to become as fast as crystallization in the bulk. This shows that the nucleation barrier becomes lower than in the bulk for $R \gtrsim 13 d$.

The critical nucleus size is determined from the observed probabilities for growth and shrinkage of nuclei as a function of nucleus size. This is based on tracking the nuclei over time depending on their spatial overlap and their average $\hat{q}_{6}$ vector, as outlined in section 2. Each nucleus is marked as growing, shrinking, or keeping its size by comparison with its immediate successor at the following time step of the measurement. A size histogram is then generated for all nuclei, and for all $N_{i}$ nuclei in a size range $\left[M_{i}-\Delta M_{i} / 2, M_{i}+\right.$ $\Delta M_{i} / 2$ ], the probability for growth (shrinkage) is simply obtained by dividing the number of growing (shrinking) nuclei by $N_{i}$. The bin sizes, $\Delta M_{i}$, for the number of particles in the nuclei are varied with the average size, $M_{i}$, such that the considered size range contains $\geq 300$ nuclei. Figure 9 shows the size dependence of the probabilities obtained for a 

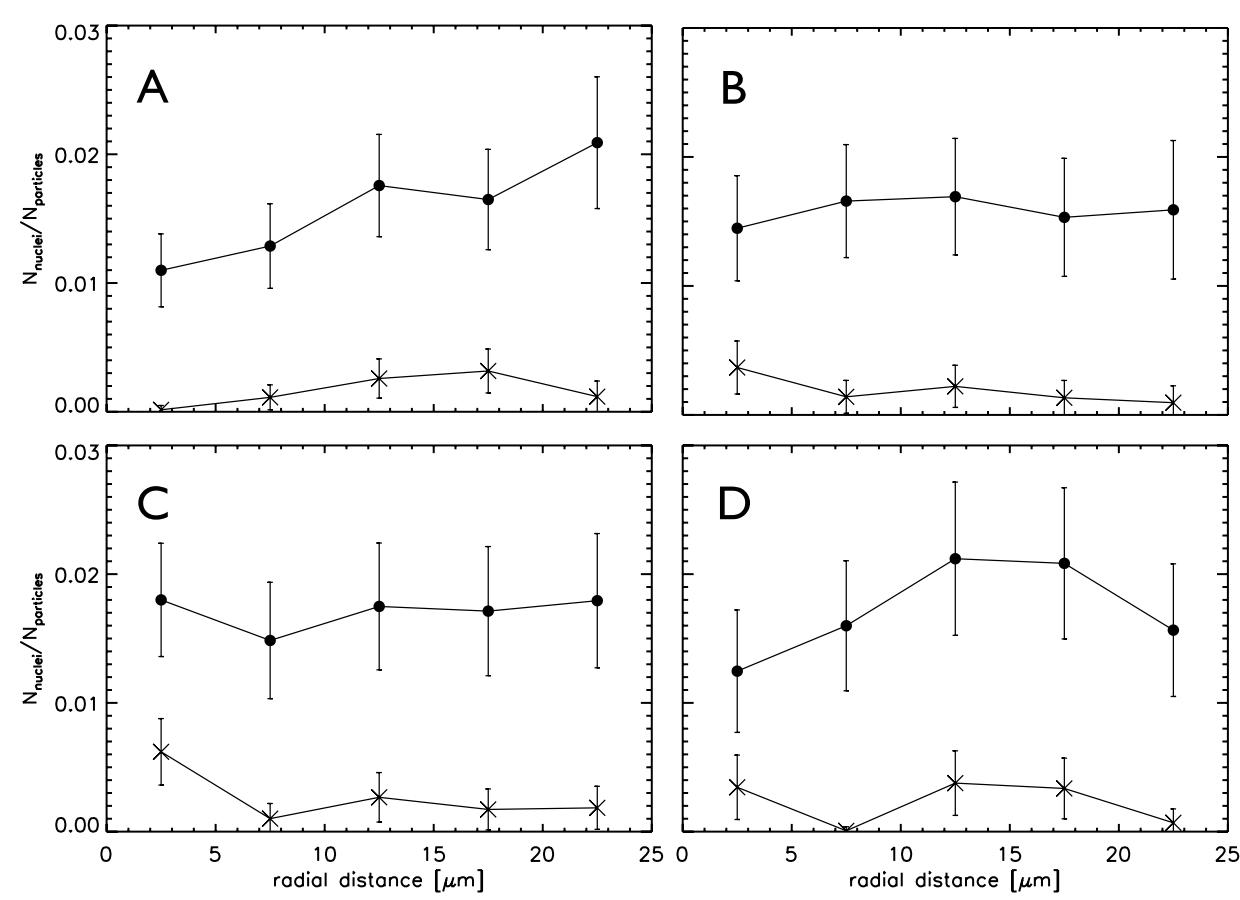

Figure 8. Number of precritical crystal nuclei normalized by the total number of particles versus distance from curved surfaces. The symbols represent the size range of the nuclei defined by the number of particles, $M$, in a nucleus: $(\bullet) M<15$ and $(\times) M \geq 15$. The radii of curvature and the volume fractions are (A) $R=15.3 \mu \mathrm{m}, \phi=0.29$; (B) $R=25.5 \mu \mathrm{m}, \phi=0.28$; (C) $R=29.8 \mu \mathrm{m}, \phi=0.31$;

(D) $R=62.5 \mu \mathrm{m}, \phi=0.24$.

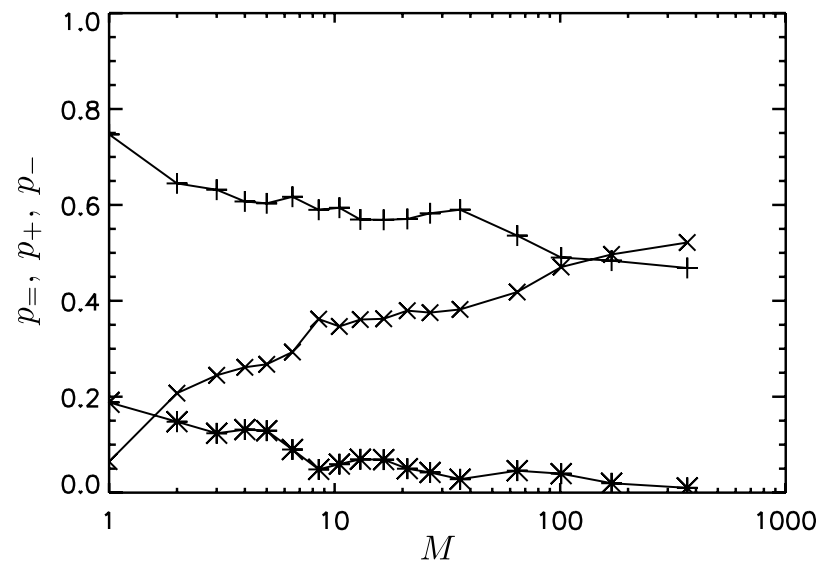

Figure 9. Probabilities for shrinkage, $p_{-}(+)$, growth, $p_{+}(\times)$, and constant size, $p_{=}(*)$, of crystal nuclei at $\phi=0.29$ as a function of nucleus size, $M$, given by the number of particles in the nucleus. Each data point corresponds to an average over $\geq 300$ nuclei observed during a measurement.

measurement at $\phi=0.29$. An estimate of the critical size, $M^{*}$, is obtained from the size at which the probabilities for growth and shrinkage cross or are equal. We find $M^{*}=800 \pm 200$ at $\phi=0.2$ and $M^{*}=150 \pm 70$ at $\phi=0.29$. Within the accuracy of the experiments, no decrease of $M^{*}$ close to the curved surfaces is found compared to the bulk. The observed suppression of crystal growth near surfaces with $R \lesssim 10 d$, however, suggests that $M^{*}$ becomes much larger in this range of $R$.

According to classical nucleation theory (CNT) a decrease of $M^{*}$ is expected for heterogeneous nucleation. For heterogeneous nucleation on a flat surface, $M^{*}$ is reduced by the factor $f(\theta)=\left[2-3 \cos (\theta)+\cos ^{3}(\theta)\right] / 4$, where $\theta$ is the contact angle of the crystal on the seed. $\Delta G_{\text {het }}^{\mathrm{CNT}}$ is reduced by the same factor with respect to $\Delta G_{\text {hom }}^{\mathrm{CNT}}$ [32]:

$$
\Delta G_{\text {het }}^{\mathrm{CNT}}=\Delta G_{\mathrm{hom}}^{\mathrm{CNT}} f(\theta) .
$$

Therefore, the shape of the barrier and the radius of a spherical critical nucleus, $r^{*}$, is the same for homogeneous nucleation and heterogeneous nucleation on a flat wall. The change of the critical volume is given by $f(\theta)$, which varies between zero and one with $\theta$. For heterogeneous nucleation on a curved seed surface, the calculation of $\Delta G_{\text {het }}^{\mathrm{CNT}}$ is much more complicated, but a result for the barrier height $\Delta G_{\text {het }}^{* \mathrm{CNT}}$ can be given [33]:

$$
\begin{aligned}
& \Delta G_{\text {het }}^{* \mathrm{CNT}}=\Delta G_{\text {hom }}^{* \mathrm{CNT}} f(\theta, x) \\
& f(\theta, x)= \frac{1}{2}\left\{1+\left(\frac{1-m x}{g}\right)^{3}\right. \\
&+x^{3}\left[2-3\left(\frac{x-m}{g}\right)+\left(\frac{x-m}{g}\right)^{3}\right] \\
&\left.+3 m x^{2}\left(\frac{x-m}{g}-1\right)\right\} \\
& g=\left(1+x^{2}-2 m x\right)^{\frac{1}{2}}
\end{aligned}
$$

where $m=\cos (\theta)$ and $x=R / r^{*}$ with the radius of the critical nucleus given by $r^{*}=2 \gamma_{\mathrm{cf}} /\left(n_{\mathrm{c}} \Delta \mu\right)$, the same value as for homogeneous nucleation with $n_{\mathrm{c}}$ the particle number density in the crystalline state. As for the flat seed, $f(\theta, x)$ varies between zero and one for contact angles $\theta<180^{\circ}$, and 


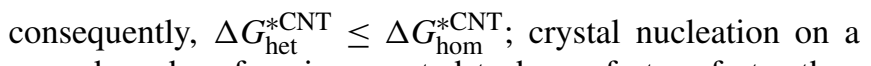
curved seed surface is expected to be as fast or faster than in the bulk according to CNT.

The observation of fast crystallization on a flat surface and the tendency of the crystal to wet curved surfaces with $R \gtrsim 15 d$ shows that $\theta$ is expected to be close to $0^{\circ}$ in our colloidal system, certainly $\theta<180^{\circ}$. For our system, $\mathrm{CNT}$ predicts a reduced barrier for heterogeneous nucleation compared to the homogeneous case. Therefore, the strong suppression of crystal growth close to the seed surface observed for $R \lesssim 10 d$ goes beyond CNT and is most probably due to an incompatibility of the crystal structure and a strongly curved surface, an effect that is not taken into account by CNT. This implies that $\gamma_{\mathrm{cs}}$ and $\theta$ depend on $R$. We expect $\gamma_{\mathrm{cs}}$ to increase for $R \lesssim 10 d$ and $\theta$ to become undefined, as $\cos (\theta)=\left(\gamma_{\mathrm{fs}}-\gamma_{\mathrm{cs}}\right) / \gamma_{\mathrm{cf}}[32]$ decreases and becomes $<-1$ for $R \sim 7 d$.

A direct measurement of $\theta$ would give valuable information about the barrier for heterogeneous nucleation. However, the very irregular shapes and rough surfaces of the observed nuclei do not allow an estimate of $\theta$ from direct observation of the crystal surface touching the seed surface. For this reason, the contact angle is not observed directly, but we expect it to depend on $R$, as mentioned above.

To compare our results with other work, we make a rough estimate of the nucleation rate density, $J$, using the volume, $V=7.5 \times 10^{-14} \mathrm{~m}^{3}$, imaged in the measurements and the time, $t^{*} \approx(1 \pm 0.3) \times 10^{5} \mathrm{~s}$, it takes for a nucleus of critical size to appear at $\phi=0.2$. We obtain the estimate $J=1 /\left(V t^{*}\right) \approx$ $(1.2 \pm 0.5) \times 10^{8} \mathrm{~m}^{-3} \mathrm{~s}^{-1}=(2.0 \pm 0.8) \times 10^{-7} D_{0} d^{-5}$, where $D_{0} \approx 1.5 \times 10^{-14} \mathrm{~m}^{2} \mathrm{~s}^{-1}$ is the free diffusion constant of the particles. This value for $J$ is at least one order of magnitude larger than that obtained in a simulation study of weakly charged Yukawa spheres with contact value $B=20 k_{\mathrm{B}} T$ and $\kappa d=5$ [34]. Nucleation rate densities of charged particles with a Yukawa-like interaction have also been determined in other experimental work [35, 36]. An increased nucleation rate close to curved surfaces, as suggested in [5] for hard spheres, is neither ruled out nor confirmed by our measurements. An increased number of crystal-like particles is found in some measurements with $6 d \lesssim R \lesssim 7 d$ at a distance $\sim 8 d$ from the surface, while other measurements do not show a peak at this distance. Also, we do not obtain any information about the line tension $\tau$ (equation (2)) from our data. $\tau$ may be too small to be measured. In fact, it is known to be small for hard spheres [31].

\section{Conclusions}

Our study of the heterogeneous and homogeneous crystal nucleation and growth of charged PMMA spheres shows that curved surfaces with a radius of curvature $R \lesssim 10 \mathrm{~d}$ hinder crystallization close to the surface, where $d$ is the particle diameter. The number of crystal-like particles is reduced within a distance $\sim 5 d$ from the surface; this distance roughly corresponds to the relaxation length of the supercooled fluid. The number of precritical crystal nuclei is found to be influenced within the same distance range $\sim 5 d$ : the number of precritical nuclei consisting of $M \geq 15$ particles is reduced, while the number of smaller nuclei is found to be of the same order as for homogeneous nucleation irrespective of the radius of curvature. This suggests that very small nuclei with a typical size smaller than the radius of curvature are not affected by the curved surface, while larger nuclei are suppressed, because they stretch out over a larger surface and are affected by the curvature. This effect implies an increase of $\gamma_{\text {cs }}$ with decreasing $R$ and goes beyond CNT, which assumes the surface tensions and the contact angle, $\theta$, to be fixed parameters of the involved materials. No direct measurement of $\theta$ could be obtained, as the contact between crystal and seed is not well defined in our measurements. However, $\theta$ is found to be close to $0^{\circ}$ on a flat wall and on seeds with curvatures $R \gtrsim 15 d$, while the observed suppression of crystal growth for curvatures $R \lesssim 10 d$ suggests that $\theta$ is not defined, as $\cos \theta<$ -1 . Precritical crystal nuclei are oriented to the curved surface for all studied $R$, even for $R \lesssim 10 d$, as hexagonal planes locally start to form parallel to the surface, as expected from the layering of the fluid near to the seed surface.

\section{Acknowledgments}

The authors acknowledge financial support by the 'Eliteprogramm für Postdoktorandinnen und Postdoktoranden der Landesstiftung Baden-Württemberg gGmbH' and the DFG through program SPP 1296.

\section{Appendix}

Crystals of hard spheres or slightly charged spheres grow as random hexagonal close packed (rhcp) crystals, which are defined by hexagonal particle planes stacked randomly according to the face centered cubic (fcc) or the hexagonal close packed (hcp) structure. This rhcp structure forms as the free energy difference between fcc and hcp is small and the ground state structure, fcc in the case of hard spheres, is reached only after very long times [37-39]. As the crystal nucleates and grows, the crystalline structure is not well defined at the crystal-fluid interface, and the orientation of the hexagonal planes is not obvious in crystal nuclei of precritical and critical size. Shortest path rings provide a way to identify hexagonal arrangements of particles and to determine their orientation from measured particle positions using topological criteria only. For each particle, shortest path rings of six particles (6-rings) are identified among the nearest neighbors, as explained in section 3.2 and in [28-30]. As disturbed crystal structures are not the exception but the usual case for measurements of small crystal nuclei, the criteria listed below are applied in addition to those given in section 3.2 to find that shortest path 6-rings are related to hexagonal particle planes. The 6-rings around a central particle with 11, 12, or 13 nearest neighbors are determined. The rules listed apply to the nearest neighbors and the bonds among them.

- The 6-ring separates the remaining nearest neighbors. These remaining particles form clusters according to their 
bonds. There must be two clusters. There is a 2-particle and a 3-particle cluster in the case of 11 nearest neighbors, two 3 -particle clusters for 12 nearest neighbors, and a 3-particle and a 4-particle cluster for 13 nearest neighbors.

As the method becomes uncertain for highly distorted structures, a 6-ring is disregarded if more than one of the following deviations from a regular fcc or hcp surrounding occurs:

- There is a particle of the 6-ring that has no bond connecting it to a particle outside the 6-ring.

- In the case of 11 nearest neighbors, two neighboring particles within the 6-ring are allowed not to have bonds to one group of particles outside the 6-ring. If there are more missing bonds from the 6-ring to the particle groups outside the 6-ring, this is counted as a deviation.

- There is a particle outside the 6-ring that has no bond to a particle in the 6-ring.

- There is at least one particle outside the 6-ring that has only one bond to its group of particles outside the 6-ring. For 11 nearest neighbors, this criterion does not apply for the 2-particle group next to the 6-ring.

In addition to the mentioned cases with 11,12 , and 13 nearest neighbors, a special case with nine nearest neighbors is taken into account, which corresponds to a fcc or hcp particle sitting on a surface, such that three nearest neighbors are missing.

- A particle with nine nearest neighbors, a 6-ring, only one group of three particles not belonging to the 6-ring, and none of the deviations listed above is taken into account in the search for particle planes with hexagonal order.

\section{References}

[1] ten Wolde P R, Ruiz-Montero M J and Frenkel D 1995 Numerical evidence for bcc ordering at the surface of a critical fcc nucleus Phys. Rev. Lett. 75 2714-7

[2] Gasser U, Weeks E R, Schofield A, Pusey P N and Weitz D A 2001 Real-space imaging of nucleation and growth in colloidal crystallization Science 292 258-62

[3] Kelton K F 1991 Crystal nucleation in liquids and glasses Solid State Physics: Advances in Research and Applications vol 45, ed H Ehrenbach and D Turnbull (Boston: Academic) pp 75-177

[4] Auer S and Frenkel D 2003 Line tension controls wall-induced crystal nucleation in hard-sphere colloids Phys. Rev. Lett. 91015703

[5] Cacciuto A, Auer S and Frenkel D 2004 Onset of heterogeneous crystal nucleation in colloidal suspensions Nature 428 404-6

[6] Gasser U 2009 Crystallization in three- and two-dimensional colloidal suspensions J. Phys.: Condens. Matter 21203101

[7] Dillmann P, Maret G and Keim P 2008 Polycrystalline solidification in a quenched $2 \mathrm{~d}$ colloidal system $J$. Phys.: Condens. Matter 20404216

[8] Dinsmore A D, Weeks E R, Prasad V, Levitt A C and Weitz D A 2001 Three-dimensional confocal microscopy of colloids Appl. Opt. 40 4152-9

[9] Prasad V, Semwogerere D and Weeks E R 2007 Confocal microscopy of colloids J. Phys.: Condens. Matter 19113102
[10] Antl L, Goodwin J W, Hill R D, Ottewill R H, Owens S M and Papworth S 1986 The preparation of poly(methyl methacrylate) lattices in non-aqueous media Colloids Surf. 17 67-78

[11] Bosma G, Pathmamanoharan C, de Hoog E H A, Kegel W K, van Blaaderen A and Lekkerkerker H N W 2002 Preparation of monodisperse, fluorescent PMMA-latex colloids by dispersion polymerization $J$. Colloid Interface Sci. 245 292-300

[12] Provencher S W 1982 A constrained regularization method for inverting data represented by linear algebraic or integral equations Comput. Phys. Commun. 27 213-27

[13] Hynninen A-P and Dijkstra M 2003 Phase diagrams of hard-core repulsive Yukawa particles Phys. Rev. E 68021407

[14] Reinke D, Stark H, von Grunberg H-H, Schofield A B, Maret G and Gasser U 2007 Noncentral forces in crystals of charged colloids Phys. Rev. Lett. 98038301

[15] Crocker J C and Grier D G 1996 Methods of digital video microscopy for colloidal studies J. Colloid Interface Sci. 179 298-310

[16] Steinhardt P J, Nelson D R and Ronchetti M 1983 Bond-orientational order in liquids and glasses Phys. Rev. B 28 784-805

[17] ten Wolde P R, Ruiz-Montero M J and Frenkel D 1996 Numerical calculation of the rate of crystal nucleation in a Lennard-Jones system at moderate undercooling J. Chem. Phys. 104 9932-47

[18] Gasser U, Schofield A and Weitz D A 2003 Local order in a supercooled colloidal fluid observed by confocal microscopy J. Phys.: Condens. Matter 15 S375-80

[19] Kashchiev D 2000 Nucleation: Basic Theory with Applications (Oxford: Butterworth-Heinemann)

[20] Auer S and Frenkel D 2001 Suppression of crystal nucleation in polydisperse colloids due to increase of the surface free energy Nature 413 711-3

[21] Martin S, Bryant G and van Megen W 2003 Observation of a smecticlike crystalline structure in polydisperse colloids Phys. Rev. Lett. 90255702

[22] Martin S, Bryant G and van Megen W 2005 Crystallization kinetics of polydisperse colloidal hard spheres. II. Binary mixtures Phys. Rev. E 71021404

[23] Sanz E, Valeriani C, Zaccarelli E, Poon W C K, Pusey P N and Cates M E 2011 Crystallization mechanism of hard sphere glasses Phys. Rev. Lett. 106215701

[24] Wessels P P F, Schmidt M and Lowen H 2004 The contact angle of the colloidal liquid-gas interface and a hard wall J. Phys.: Condens. Matter 16 S4169-84

[25] Pusey P N and van Megen W 1986 Phase behaviour of concentrated suspensions of nearly hard colloidal spheres Nature 320 340-2

[26] Pusey P N, van Megen W, Bartlett P, Ackerson B J, Rarity J G and Underwood S M 1989 Structure of crystals of hard colloidal spheres Phys. Rev. Lett. 63 2753-6

[27] Auer S and Frenkel D 2001 Prediction of absolute crystal-nucleation rate in hard-sphere colloids Nature 409 1020-3

[28] Franzblau D S 1991 Computation of ring statistics for network models of solids Phys. Rev. B 44 4925-30

[29] O'Malley B 2001 Molecular dynamics investigation of crystallization in the hard sphere system $P h D$ Thesis Royal Melbourne Institute of Technology

[30] O'Malley B and Snook I 2005 Structure of hard-sphere fluid and precursor structures to crystallization J. Chem. Phys. 123054511

[31] Cacciuto A, Auer S and Frenkel D 2004 Breakdown of classical nucleation theory near isostructural phase transitions Phys. Rev. Lett. 93166105

[32] Turnbull D 1950 Kinetics of heterogeneous nucleation J. Chem. Phys. 18 198-203 
[33] Fletcher N H 1958 Size effect in heterogeneous nucleation J. Chem. Phys. 29 572-6

[34] Auer S and Frenkel D 2002 Crystallization of weakly charged colloidal spheres: a numerical study J. Phys.: Condens. Matter 14 7667-80

[35] Wette P, Schope H J, Liu J and Palberg T 2003 Solidification in model systems of spherical particles with density-dependent interactions Europhys. Lett. 64 124-30

[36] Engelbrecht A, Meneses R and Schope H J 2011 Heterogeneous and homogeneous crystal nucleation in a colloidal model system of charged spheres at low metastabilities Soft Matter 7 5685-90

[37] Woodcock L V 1997 Entropy difference between the face-centered cubic and hexagonal close-packed crystal structures Nature 385 141-3

[38] Bolhuis P and Frenkel D 1997 Tracing the phase boundaries of hard spherocylinders J. Chem. Phys. 106 666-87

[39] Pronk S and Frenkel D 1999 Can stacking faults in hard-sphere crystals anneal out spontaneously? J. Chem. Phys. 110 4589-92 